\section{Mehr leukämiekranke Kinder in AKW-Nähe}

\begin{abstract}
Weniger als $5 \mathrm{~km}$ von einem Kernkraftwerk entfernt zu leben, erhöht einer französischen Studie zufolge das Leukämierisiko von Kindern. Allerdings lässt sich der Anstieg des Risikos nicht durch radioaktive Emissionen erklären.
\end{abstract}

D. ie jetzt publizierten Daten der Geocap-Studie beziehen sich auf die Jahre 2002 bis 2007. In dieser Zeit wurde in Frankreich bei 2.753 Kindern im Alter unter 15 Jahren eine Leukämie diagnostiziert. Das Zuhause von 99 Kindern lag in einem Radius von $20 \mathrm{~km}$ um ein Atomkraftwerk. 14 leukämiekranke Kinder lebten innerhalb der 5-km-Zone. Damit war, bezogen auf eine repräsentative Stichprobe von 30.000 Kindern, die Leukämierate im innersten Zirkel signifikant höher als in einer Entfernung von $20 \mathrm{~km}$ oder mehr (Odds Ratio 1,9). Welcher Altersgruppe die Kinder angehörten $(<5,4-9$ oder 10-14 Jahre), machte dabei keinen Unterschied.

$\mathrm{Zu}$ demselben Ergebnis kommt eine zeitgleich durchgeführte Inzidenzstudie:
In der 5-km-Zone war die Zahl registrierter Leukämiefälle signifikant größer als die der erwarteten Fälle (14 vs. 7,4). Überraschenderweise zeigte sich jedoch keinerlei Zusammenhang zwischen der Leukämierate und den radioaktiven Emissionen der AKW. Gebiete mit höherer Strahlenbelastung wiesen keine erhöhten Erkrankungsraten auf. Möglicherweise, so die Autoren, sind andere Faktoren in der Umgebung des AKWs für den Risikoanstieg verantwortlich.

Das Studienergebnis steht im Widerspruch zu einer vorausgegangenen Studie, in der die Leukämieinzidenz in den Jahren 1990 bis 2001 analysiert worden war. In diesem Zeitraum waren in der näheren Umgebung von französischen AKWs nicht mehr Kinder an Leukämie erkrankt als in größerer Distanz oder als erwartet. Das war auch dann nicht der Fall, wenn die gesamte Periode von 1990 bis 2007 überblickt wurde. Während dieser 18 Jahre war auch kein Anstieg der Leukämierate zu beobachten.

Fazit: Auch sonst ist die Datenlage widersprüchlich: Mehrere epidemiologische Studien, z.B. aus der Schweiz, Frankreich oder Finnland, haben landesweit keine stärkere Leukämiegefährdung erkennen lassen. In der Umgebung einzelner Kernkraftwerke, z. B. Krümmel oder Sellafield, wurden aber sehr wohl erhöhte Erkrankungsraten dokumentiert. Und in der deutschen KiKK-Studie wurde bei Kindern unter fünf Jahren, die im Umkreis von $5 \mathrm{~km}$ um ein AKW lebten, eine Verdopplung des Leukämierisikos festgestellt. Beate Schumacher

Sermage-Faure $C$ et al. Childhood leukemia around French nuclear power plants-The Geocap study, 2002-2007. Int J Cancer. 2012;131(5): E769-80.

\title{
Kinder mit Krebs - geheilt, aber nicht gesund
}

\section{Nach der Heilung von Kindern mit Krebs ist bei einem Teil der kleinen Patienten noch nicht alles überstanden. Viele Jahre später kann es erneut zu Krebserkrankungen kommen, die sich vom Primärtumor unterscheiden.}

W erden krebskranke Kinder durch Chemotherapie und Bestrahlung geheilt, besteht Jahrzehnte später ein erhöhtes Risiko für Darmkrebs. In mehreren Studien wurde eine kumulative Inzidenz dieses Sekundärtumors zwischen $0,1 \%$ und 2,4\% im Alter von 30 Jahren dokumentiert. Primärtumoren im Kindesalter waren etwa hämatologische Malignome, Hirntumoren und Sarkome.

Pädiater der St. Jude-Kinderklinik in Memphis untersuchten jetzt in einer Fall-Kontroll-Studie, wie oft Darmkrebs viele Jahre nach der Heilung des Primärtumors auftritt und wie dies mit der Bestrahlung und der Chemotherapie des Primärtumors zusammenhängt. Dazu werteten sie die Daten von über 13.000 Patienten aus, die als Kinder an der Klinik eine Krebstherapie erhalten hatten.

Insgesamt 19 Patienten entwickelten ein Adenokarzinom des Kolons oder des Rektums. Das häufigste Primärmalig- nom war bei ihnen ein Hodgkin-Lymphom, an dem neun Patienten als Kinder erkrankt waren. Im Median waren die Kinder zum Zeitpunkt der Diagnose des Primärtumors zehn Jahre, zum Zeitpunkt der Diagnose des Sekundärtumors 33 Jahre alt - der jüngste Patient 11, der älteste 47. Außer bei einem Patienten, der nur die Chemotherapie erhalten hatte, wurden zur Krebsbehandlung Radiatio und Chemotherapie kombiniert.

In der US-Studie lag die kumulative Inzidenz für ein Kolorektalkarzinom über einen Zeitraum von 40 Jahren seit der Diagnose des Primärtumors bei 1,4\%. Im Vergleich zur Allgemeinbevölkerung hatten Patienten, die Krebs in der Kindheit überlebten, ein um das 11-Fache erhöhtes Darmkrebs-Risiko. Bei Patienten, die jünger als 40 Jahre waren, war das Risiko etwas höher (11,3fach), bei Patienten die älter als 40 Jahre waren, etwas niedriger (10,3-fach).
Die statistische Auswertung ergab zudem, dass die Verwendung von Alkylanzien in der Chemotherapie das Risiko für ein späteres kolorektales Karzinom um das 8,8-Fache erhöhte. Und: Mit jeder Steigerung der Bestrahlungsdosis um 10 Gy stieg das Risiko für den Sekundärtumor im Darm um 70 \% (Odds Ratio 1,7; 95\%iges Konfidenzintervall von 1,2 bis $2,5 ; \mathrm{p}=0,003)$. Erstmals konnten die USÄrzte belegen, dass das Risiko für den Sekundärtumor umso höher war, je mehr Darmabschnitte in der Kindheit der Bestrahlung ausgesetzt waren.

Fazit: Eine wichtige Erkenntnis dieser Studie ist nach Ansicht der Wissenschaftler, dass bei Patienten, die als Kinder Krebs hatten, Darmkrebs deutlich früher im Leben auftreten kann als in der übrigen Bevölkerung. Bei Patienten mit Abdominalbeschwerden und unerklärlichem Eisenmangel sollte frühzeitig geklärt werden, ob vielleicht Darmkrebs die Ursache ist.

Peter Leiner

Nottage K et al. Secondary colorectal carcinoma after childhood cancer. J Clin Oncol. 2012;30(20): 2552-8. 